\title{
Underestimating Correlation from Scatterplots
}

\author{
Robert F. Strahan and Chris J. Hansen \\ lowa State University
}

Eighty subjects estimated the correlation coefficient, $r$, for each of 13 computer-printed scatterplots. Making judgments were 46 students in a graduate-level statistics course and 34 faculty and graduate students in a department of psychology. The actual correlation values ranged from .010 to .995 , with 200 observations in each scatterplot and with the order of scatterplot presentation randomized. As predicted, subjects underestimated the degree of actual correlation. Also as predicted, but with substantial moderation by a method-of-presentation factor, this underestimation was most pronounced in the middle of the correlational range- between the 0 and 1 extremes. Though perception of correlation was shown not to be veridical (i.e., in terms of $r$ ), little support was given one alternative view-its being in terms of $r^{2}$.

Various aspects of intuitive statistical behavior have been studied (Peterson \& Beach, 1967), and a number of investigations have focused on judgments of association or relation (Beach \& Scopp, 1966; Erlick, 1966; Gray, Barnes, \& Wilkinson, 1965; Pollack, 1960, 1974; Smedslund, 1963). However, the direct estimation of product-moment correlation from scatterplots-the object of this research-appears not to have been studied.

That the correlation coefficient, $r$, assumes misleadingly large values is not infrequently

APPLIED PSYCHOLOGICAL MEASUREMENT

Vol. 2, No. 4 Fall 1978 pp. 543-550

(c) Copyright 1978 West Publishing Co. noted in elementary statistics texts. An $r$ of .30 expresses a relation in which only $9 \%\left(r^{2}=.09\right)$ of the variance of one variable is shared with that of the other; an $r$ of .70, only $49 \%$. Thus, it was expected that people would underestimate the magnitude of correlation when judging from scatterplots. Some suggestion that judgment of correlation might be in terms of $r^{2}$ rather than (or perhaps in addition to) $r$ has been given by Pollack (1960) and by Strahan (in press).

A second hypothesis was that the extent of underestimation would vary with the level of presented (actual) $r$. There should be no bias for a circular scatterplot $(r=0)$ nor for one that is a straight line $(r= \pm 1)$; it is only between these conspicuous extremes that underestimation should occur. Experience in examining scatterplots, however, suggested that until the plot becomes very nearly a straight line, rather dramatic underestimation might still occur. Methodological complexities (to be described later) unfortunately prevented precise prediction here.

\section{Method}

\section{Materials}

Booklets of 13 bivariate normal scatterplots were prepared, each plot containing 200 data points. Four different configurations of points were randomly generated by computer; and for 
any given booklet, all scatterplots had the same configuration, the individual plots differing only in level of correlation. Within each booklet the order of scatterplots was randomized.

For half the booklets, presentation or spacing of correlational level was linear in $r(r=.010$, $.092, .173, \ldots, .908, .990)$. For the other half, the presentation was linear in $r^{2}\left(r^{2}=.010\right.$, $.092, \ldots$, and thus here $r=.100, .303$, $.416, \ldots, .953, .995)$. It was suspected that despite instructions warning against subject presuppositions, subjects might nevertheless expect the correlational values to be equally spaced and might base their estimates to some extent on that presumed regularity. Thus, provision was made for the investigation of this anticipated context or method factor. Presentation equally spaced in $r$ was a natural choice (with the number of levels chosen to minimize values likely to be popular a priori-e.g., .20, .30, .40). Presentation equally spaced in $r^{2}$ was consistent with interest in whether subjects perceive scatterplots more in accordance with proportion of shared variance than in terms of $r$.

The four configurations and two modes of presentation generated eight different scatterplot booklets. The plots themselves were reproduced on $81 / 2$ " by 11 " sheets of plain white paper. Black lines without interval marks served as horizontal and vertical axes. Black dots indicated a single bivariate data point, and numerals signified the frequency at a single printed space when the observations there (or very near there) were greater than one in number. The variances of the two variables were equal, and the units of the abscissa and ordinate were of equal length.

\section{Subjects and Procedure}

A Statistics Class sample consisted of students enrolled in an advanced basic statistics course taught by the first author. These were predominantly graduate students who, for the most part, had had previous statistics experience; they were mostly in psychology, child development, educa. tion, and related disciplines.
These subjects were given the scatterplot booklets during a regular class period. A cover sheet requested that students simply look at each scatterplot, then write the best estimate of the correlation coefficient, using two decimal places (even though there might be very little faith in the accuracy of the second figure). Independent work was called for, without recourse to books or to other persons. Students were cautioned against assuming that a particular range of correlational values was necessarily covered or that the correlational values were necessarily ordered or in equal steps. They were told that different sets of scatterplots had been distributed and that they should make their estimates simply on the basis of the particular plots they had received. In addition to this cover sheet information, students were reminded orally that a correlation coefficient ranges between 1 and -1 (lectures on correlation had been given some few weeks prior to data collection). Of about 55 students present, 46 provided usable protocols, i.e., complete data estimates that satisfied a gross ordinality criterion. ${ }^{1}$

A second sample of subjects was comprised of Psychology Department faculty and post firstyear graduate students at Iowa State University (first-year graduate students were largely represented in the first sample) ${ }^{2}$. Scatterplot booklets

\footnotetext{
'Although in the presented-by- $r$ case a difference between correlations of only one step (an $r$ difference of .082 ) is not readily discernible with the order of presentation randomized, a difference of four steps $(\Delta r=.328)$ is typically quite apparent. There are nine 4-step differences in the 13 scatterplots; and the number of disordinal judgments, or reversals, was recorded. Three potential subjects made at least three such reversals, and their data were removed from the study. For no qualifying subject were there more than two reversals.

${ }^{2}$ An undetermined but large number of these faculty and
students had participated the previous year in a related esti-
mation task (Strahan, in press) and had received a brief re-
port of that study's outcome. To the extent that that experi-
ence was related to the present work, however, it would seem
to have been a factor working against rather than for the
substantive hypotheses.
} 
with previously described cover sheets were distributed in subjects' mailboxes and collected over the ensuing few weeks. To half the scatterplot booklets was added the cover sheet statement that all correlations were non-negative. Of the 29 faculty and 26 graduate students to whom booklets were given, 19 faculty and 15 students returned usable materials. Three other protocols were discarded for previously noted reasons.

\section{Analyses}

The basic dependent variable was $r_{e}-r_{p}$, the algebraic difference between estimated and presented correlational values. Analyses of variance (Anovas) were performed separately for the four groups generated by the distinctions presentedby- $r$ versus presented-by- $r^{2}$ and Statistics Class versus Psychology Department. These were $4 \times$ 13 split-plot analyses, the fixed factors ${ }^{3}$ respec: tively being Configuration (between groups) and Level (within groups). Because of the manner in which materials were distributed, cell sizes were typically not equal, and the method of unweighted-means was used.

Two additional Anovas were performed with just the Psychology Department sample; approximately half of the sample also had been instructed that there were no negative correlations. Here the analysis was a $2 \times 13$ split plot,

\footnotetext{
${ }^{3}$ Although Configuration could properly have been considered a random factor-the four particular configurations were randomly selected and the population of ultimate interest was, in fact, all possible correlational shapes-logistical considerations precluded this analytic course. Little more than four different configurations could, with relative ease and inexpensiveness, be prepared; and degrees of freedom with as few as four would necessarily be too few to provide an adequately sensitive test of the factor of principal interest-Level. Thus, Configuration was treated as a fixed factor; and inferences hold, strictly speaking, only for the particular configurations presented. However, given the representativeness that randomization affords, together with the possibility of testing whether Configuration was in fact a significant source of variation, this was not considered a serious limitation of the analysis.
}

the new between-subjects fixed factor being Instructions. Unequal cell sizes were a greater problem here because of the smaller numbers available for the cells; consequently, the exact least squares method was used rather than the unweighted-means approximation, and Configuration was ignored as a factor.

The Anovas investigated discrepancy between estimated and presented correlation as a function of Level, Configuration, and (for half the subjects) Instructions. To answer the fundamental question whether there is overall bias in the estimation of correlation, $r_{e}-r_{p}$ discrepancy scores were summed over the 13 levels and $t$-tests calculated for these overall discrepancy measures.

In the above description, $r_{e}-r_{p}$ represents the difference between the empirical estimation of correlation and a theoretical or expected value. Implicit in the expression $r_{e}-r_{p}$ is the expectation that subjects are (or at least might be) accurate perceivers of correlation-that is, judging $r$ to be close to the value of the scatterplot actually presented.

Given the possibility that subjects might perceive correlation in terms of $r^{2}$ rather than $r$, however, it became of interest to perform another, parallel set of analyses. Here the theoretical or expected correlation would be $r_{p}^{2}$ and the new dependent variable, $r_{e}-r_{p}^{2}$. A generic expression indicating the difference between estimated and theoretical correlation is $r_{e}-r_{i}$. If it is theorized that subjects accurately perceive correlation (i.e., veridically perceive $r$ as $r$ ), then $r_{t}=r_{p}$. If, on the other hand, subjects mistakenly (but accurately) perceive $r$ as $r^{2}$, then $r_{t}=r_{p}^{2}$.

\section{Results}

Graphic display of the results is given in Figures 1 and 2. For the presented-by-r and presented-by- $r^{2}$ groups separately, the mean estimated correlation, $\boldsymbol{F}_{a}$, is plotted as a function of the presented correlation, $r_{p}$, for both the Statistics Class and Psychology Department samples. The lower left-to-upper right straight line is the 
Figure 1

Relation Between Mean Estimated and Presented Correlation: Presented-by-r Samples

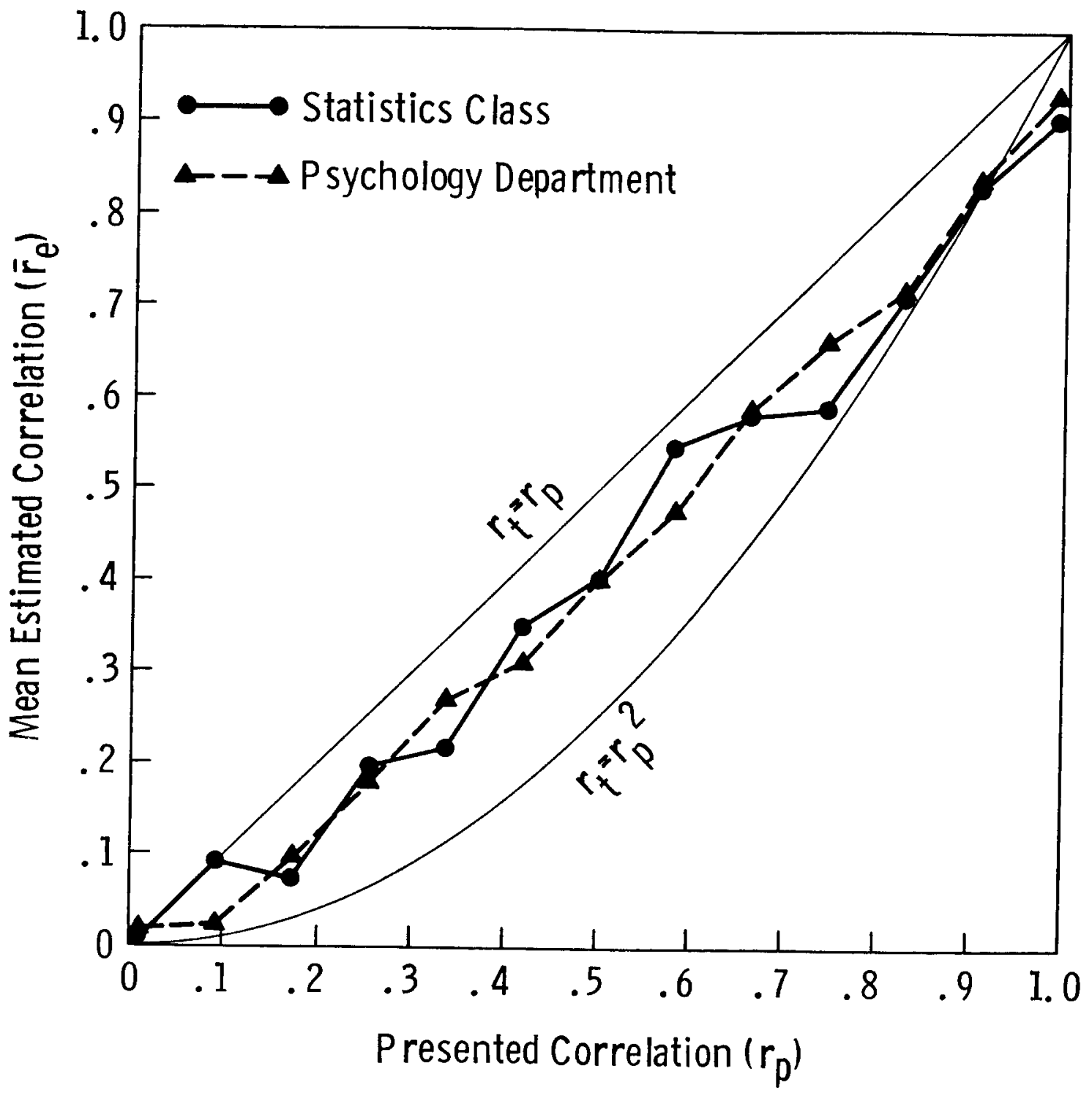


Figure 2

Relation Between Mean Estimated and Presented Correlation:

Presented-by- $\mathbf{r}^{2}$ Samples

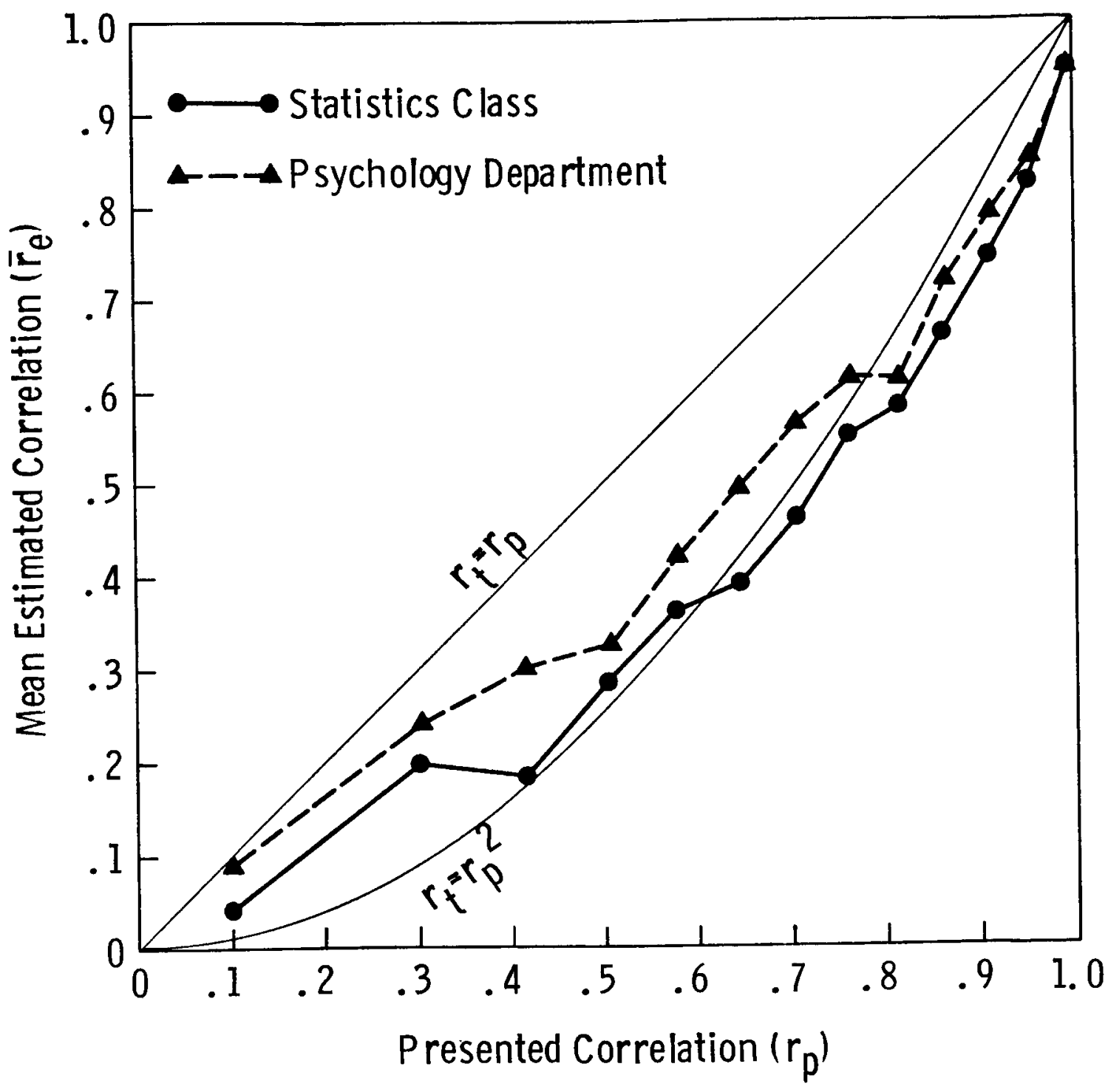


locus of points representing perfect $r$-perceivedas- $r$ estimation $\left(r_{t}=r_{p}\right)$; the bowed line, the locus of points reflecting perfect $r$-perceived-as- $r^{2}$ estimation $\left(r_{t}=r_{p}^{2}\right)$.

Subjects quite consistently underestimated in their judgments of correlation (the empirical curves are below the $r_{t}=r_{p}$ line), and significantly so as shown by $t$-tests of overall discrepancy scores. For presented-by-r subjects, $t(24)=-3.54, p<.01$ for the Statistics Class and $t(15)=-3.19, p<.01$ for the Psychology Department. Underestimation was particularly marked in the presented-by- $r^{2}$ samples, where for 37 of 39 subjects, overall discrepancy scores were less than zero. Here $t(20)=-7.95, p<.001$ for the Statistics Class; and $t(17)=-6.87, p<.001$ for the Psychology Department.

The predicted quadratic component of the Level factor's impact on the $r_{e}-r_{p}$ difference scores was apparent for the presented-by- $r^{2}$ samples, where for Statistics Class and Psychology Department subjects, $F(1,204)=95.75, p<$ .001 and $F(1,168)=50.35, p<.001$, respectively. In the presented-by-r cases, suggestion of a quadratic relation was much less striking: $F(1,252)=3.35, p<.10$ for Statistics Class subjects and $F(1,144)=4.33, p<.05$ for Psychology Department subjects.

For no analysis was either Configuration or the Level-Configuration interaction significant. Similarly, neither Instructions nor its interaction with Level proved significant in the additional Anovas performed just on Psychology Department subjects.

Turning to the question whether subjects tend to perceive $r$ as $r^{2}$ rather than $r$, the mode of scatterplot presentation had a greater influence. For presented-by- $r$ subjects, there was marked overestimation of $r_{t}=r^{2}$ : for the Statistics Class, $t(24)=3.63, p<.01$; for the Psychology Department, $t(15)=3.44, p<.01$. The Level factor was clearly an influence here, and the relation was strongly quadratic: $F(1,252)=50.62, p<.001$ and $F(1,144)=50.63, p<.001$ for the Statistics Class and Psychology Department samples, respectively. (The linear component also was signi- ficant for Statistics subjects: $F(1,252)=6.91, p<$ .01.)

By contrast, for the presented-by- $r^{2}$ subjects, overestimation was nonsignificant for the Statistics Class sample $[t(20)=-.90, p>.10]$ and only marginally significant for the Psychology Department sample $[t(17)=1.98, p<.10]$. For these as well as the previous subjects, Level had significant impact, although the nature of the relation was more complex here than in the presented-by-r case. For both Statistics Class and Psychology Department samples, there were strong linear trends: $F(1,204)=49.05, p<.001$ and $F(1,168)=84.87, p<.001$. For the former, there were also significant quadratic $(F(1,204)=$ $4.49, p<.05)$ and cubic $(F(1,204)=5.26, p<.05)$ components; for the latter, a significant cubic trend $(F(1,168)=7.97, p<.01)$.

\section{Discussion}

It is clear that subjects underestimate the degree of correlation when viewing scatterplots. Though the extent of this underestimation is a function of the mode of presentation (more pronounced discrepancies were seen in the presented-by- $r^{2}$ cases), $t$-tests were highly significant for all four independent samples.

It also appears that underestimation of correlation depends on the level of presented correlation, with greater deviations (as naturally expected) between presented $r$ extremes of 0 and 1 . This was markedly the case for the two presented-by- $r^{2}$ samples, less certainly so for the presented-by- $r$ subjects.

But the hypothesis that subjects might perceive $r$ as $r^{2}$ rather than veridically as $r$ was not well supported, though the picture was complicated again by mode of presentation. For presented-by- $r$ samples, there was clear overestimation; for presented-by- $r^{2}$ samples, nonsignificant results (though borderline for one of the two samples). In addition, level of presented $r$ was very highly significant for all four samples under the $r$-perceived-as- $r^{2}$ theory.

Because the mean estimated $r$ 's fell between the $r_{t}=r_{p}$ and $r_{t}=r^{2}$ curves, one might suspect 
that some subjects actually were following the first model; others, the second. Histograms of estimated-theoretical discrepancies, collapsing across levels of $r_{p}$, provided data contrary to this possibility; however, the distributions appeared continuous with no signs of bimodality or tendency toward bimodality.

Alternatively, it might be supposed that a natural tendency to perceive $r$ as $r^{2}$ had been tempered by an academic understanding of the misleading character of the correlation coefficient. Such a circumstance could lead to the results actually observed and be consistent as well with the non-bimodal distributions revealed by the histograms. But if this were so, it might also be expected that the Psychology Department subjects-faculty and advanced graduate students-would have reported estimated correlations closer to $r_{t}=r_{p}$ than those of the less experienced Statistics Class subjects. Though formal statistical comparisons were not made (because of differences in procedure for these two sets of subjects), graphic inspection of the results indicated no confirmation of that expectation.

On the other hand, it might be argued that not many investigators-neither experienced researchers nor neophytes-have really had much experience in examining scatterplots. (A common lament among statistical advisors is that researchers do not look at their data, but only at summary statistics.) This raises the question why subjects might be expected to follow any particular model at all in their estimation of correlation from scatterplots. Both $r$-perceived-as- $r$ and $r$-perceived-as- $r^{2}$ are models based on abstract concepts (so far as the estimation of strength of relation is concerned) having little direct physical relevance- $r$ being the slope of the (standardized) regression line, $r^{2}$ the proportion of shared variance. Thus it would seem of interest, given the results of the present study, to ask a different question: What is the metric that subjects actually use in estimating from scatterplots the strength of linear relation between two variables? Presumably, with subjects naive to the study of statistics, that metric would have to be based on the "thickness" or "thinness" of the elliptical shape of the scatterplot. Some simple models, such as the difference and the ratio between major and minor axes of that ellipse, have been compared informally with the present data; but their lack of fit is even more pronounced than that of $r_{t}=r_{p}$ and of $r_{t}=r_{p}^{2}$. Of course, this study was not directed to the issue of what metric subjects actually employ; the adequate addressing of that question requires a different experimental approach.

A number of potential limitations of the current work have been remarked on already. The entire correlational range from -1 to 1 was not presented (to preclude possible confusion with negative numbers). Subjects may naturally tend to use certain numbers more than others (e.g., .50 rather than .53 - which might explain the saw-edge nature of Figures 1 and 2). Of necessity, at high levels of $r_{p}$, numbers rather than dots were increasingly used in the computergenerated scatterplots; and it is conceivable that subjects could not appreciate the density thereby represented, thus resulting in underestimation of correlation at those high levels of $r_{p}$.

On the other hand, some diversity of sampling and method of presenting $r_{p}$ was afforded; and a consistent and clear answer emerged to the basic questions of this research: Subjects underestimate correlation when judging from scatterplots, and that underestimation is a function of the correlational level presented.

\section{References}

Beach, L. R., \& Scopp, T. S. Inferences about correlations. Psychonomic Science, 1966, 6, 253-254.

Erlick, D. E. Human estimates of statistical relatedness. Psychonomic Science, 1966, 5, 365-366.

Gray, C. W., Barnes, C. B., \& Wilkinson, E. F. The process of prediction as a function of the correlation between two-scaled variables. Psychonomic Science, 1965, 3, 231-232.

Peterson, C. R., \& Beach, L. R. Man as intuitive statistician. Psychological Bulletin, 1967, 68, 29-46. 
Pollack, I. Identification of visual correlational scat terplots. Journal of Experimental Psychology, $1960,59,351-360$.

Pollack, I. Visual perceptualization of tetrachoric correlations. In H. R. Moskowitz et al. (Eds.), Sensation and measurement. Dordrecht-Holland: D. Reidel Publishing Company, 1974.

Smedslund, J. The concept of correlation in adults. Scandinavian Journal of Psychology, 1963, 4. 165-173.

Strahan, R. F. On the nonintuitive nature of the correlation coefficient: Subjective estimation of three- variable relations. Multivariate Behavioral $\mathbf{R e}$ search, in press.

\section{Acknowledgment}

We are grateful for our colleagues' cooperation as subjects in this study.

\section{Author's Address}

Robert F. Strahan, Department of Statistics, Snedecor Hall, Iowa State University, Ames, IA 50011. 